\title{
CLINICOPATHOLOGICAL STUDY AND MANAGEMENT STRATEGIES OF LIVER ABSCESS IN A TERTIARY CARE CENTRE
}

\author{
Nitish Kumar1, Ranjit Kumar Deka², Arpan Choudhury³, Bijit Gogoi ${ }^{4}$ \\ 1Postgraduate Student, Department of Surgery, Gauhati Medical College. \\ 2Professor, Department of Surgery, Gauhati Medical College. \\ ${ }^{3}$ Postgraduate Student, Department of surgery, Gauhati Medical College. \\ ${ }^{4}$ Assistant Professor, Department of Surgery, Gauhati Medical College.
}

ABSTRACT

\section{BACKGROUND}

India being a tropical country, liver abscess is a major problem, often with lethal consequences and diagnostic/therapeutic challenges. We have described the clinical profile, microbiological aetiologies, and management outcomes in patients with liver abscess.

\section{MATERIALS AND METHODS}

During the period of the month from $01 / 07 / 2015$ to $30 / 06 / 2016$, a prospective study was performed involving 50 patients admitted to the in-patient ward of the Department of General Surgery of Gauhati Medical College and Hospital, their diagnosis was made on the basis of clinical features, laboratory investigations and radiological evidence of liver abscess.

\section{RESULTS}

Pyogenic liver abscess (PLA) was the most common (64\%) type of liver abscess among the study group. The most common aetiology of pyogenic liver abscess was Escherichia coli. Ultrasonography (USG) of the abdomen was accurate and cost effective in diagnosis of liver abscesses. Percutaneous catheter drainage (PCD) was the most effective method of treatment. Right lobe was predominantly involved in amoebic and pyogenic abscesses.

\section{CONCLUSION}

In developing countries like India, the clinical presentation of liver abscess has not varied over time. At present, rapid diagnosis and image-guided percutaneous drainage offer a better prognosis for liver abscess. Appropriate use of minimally invasive drainage techniques reduces mortality. We suggest early recognition of clinical features and prompt abdominal USG as cost-effective means for treatment initiation and reducing complications.

\section{KEYWORDS}

Liver Abscess.

HOW TO CITE THIS ARTICLE: Kumar N, Deka RK, Choudhury A, et al. Clinicopathological study and management strategies of liver abscess in a tertiary care centre. J. Evolution Med. Dent. Sci. 2017;6(19):1501-1504, DOI: 10.14260/Jemds/2017/329

\section{BACKGROUND}

Liver abscess (LA) is defined as collection of purulent material in liver parenchyma which can be due to bacterial, parasitic, fungal, or mixed infection. Liver abscess is a common condition in India. India has $2^{\text {nd }}$ highest incidence of liver abscess in the world. ${ }^{1}$ In tropical country like India, both amoebic and pyogenic liver abscess (PLA) continue to be an important cause of morbidity and mortality. ${ }^{1}$

Out of total incidence of LA, approximately two-thirds of cases in developing countries are of amoebic (ALA) aetiology and three-fourths of cases in developed countries are pyogenic. ${ }^{1}$

PLA result from ascending biliary tract infection, haematogenous spread through portal venous system, septicaemia with involvement of liver by way of hepatic arterial circulation and secondary spread from

Financial or Other, Competing Interest: None.

Submission 02-01-2017, Peer Review 08-02-2017,

Acceptance 14-02-2017, Published 06-03-2017.

Corresponding Author:

Dr. Ranjit Kumar Deka

House No. 3, Hill Side,

Kushal Konwar Road

Guwahati-781003, Assam, India.

E-mail: ranjit_deka@yahoo.co.uk

DOI: $10.14260 /$ jemds $/ 2017 / 329$ intraperitoneal infection. Escherichia coli, Klebsiella, and Streptococcus are the most common aetiology of PLA.

Although no distinct clinical criteria exist for distinguishing ALA and PLA, the differential diagnosis can be made based on the following criteria- younger age, resident, or recent travel to areas of endemic amoebiasis, diarrhoea, and marked abdominal pain raise clinical suspicion of ALA. The diagnosis is confirmed by ultrasonography (USG), serological tests such as indirect haemagglutination test or ELISA (Enzyme linked immunosorbent assay), reddish brown (Anchovy paste like material) aspirate from the abscess, negative Gram stain, rapid resolution after Metronidazole treatment. The diagnosis of PLA is based on picket fence configuration of temperature chart, nausea, vomiting, anorexia, haematological analysis of leukocytosis, anaemia, and positive blood or aspirate culture for bacterial aetiology. The treatment of liver abscesses has evolved remarkably with minimal invasive drainage taking the centre stage. Radiological imaging has improved diagnostic competence and has altered therapeutic strategy by allowing the possibility of percutaneous approach using needle aspiration or catheter drainage while open surgery should be reserved for management of complicated cases.

In view of these recent developments, a prospective clinical study was undertaken at the Gauhati Medical College and Hospital, Guwahati, under Srimanta Sankaradeva 
University of Health Sciences to analyse in-depth, regarding the different clinical behaviour of liver abscess in relation to age, sex, aetiologies, locations and diagnostic and management strategies of liver abscess.

\section{Aims and Objectives}

The objective is to study a minimum of 50 cases of Liver Abscesses and to determine

- Demographic profile (Age, Sex).

- Aetiology.

- Spectrum of Clinical Presentations.

- Evaluate laboratory investigation profile.

- The efficacy of Ultrasonography and other radiological studies in determining the aetiology of liver abscess and in differentiating it from other hepatobiliary conditions which mandate change in the treatment.

- $\quad$ To study the bacteriological characteristics including its antibiotic sensitivity.

- Amoebic Serology Tests Using ELISA.

- To evaluate efficacy, complications, morbidity \& mortality, duration of hospital stay associated with Management Strategies followed-

1. Conservative Therapy (Antibiotics, metronidazole etc.).

2. Sonographic-guided percutaneous drainage (Percutaneous Aspiration or Percutaneous Pigtail Catheter Drainage) + Antibiotics Coverage.

3. Open surgical drainage.

\section{MATERIALS AND METHODS}

The present study was conducted at Gauhati Medical College and Hospital during the period from 01-07-2015 to 30-062016.

Institutional approval of the study protocol was obtained and the patients included in the study were informed about the proposed study and informed consent was obtained from each patient.

\section{Inclusion Criteria}

1. All cases of liver abscess diagnosed clinically and ultrasonographically.

2. All cases of bacterial and parasitic liver abscess.

3. All cases in evolving, liquefied \& ruptured stage with or without peritonitis.

4. All cases of clinical liver abscess with elevated total leucocyte count (TLC), LFT, and serologically amoebic antigen positive.

\section{Exclusion Criteria}

1. Traumatic Liver Abscess.

2. Past history of liver abscess.

3. Associated with malignancy.

The diagnosis of liver abscess was made based on history, clinical features, laboratory investigations, radiology, serological investigations, blood culture, and culture from the aspirate. Patients were treated with medical treatment with or without one of the following-percutaneous needle aspiration, percutaneous catheter drainage or open surgical drainage. Cure was defined as improvement clinically with subsidence of fever, and local signs, symptoms, decrease in
WBC count and follow-up ultrasonography showed reduction in size $<3 \mathrm{~cm}$ in diameter and no evidence of relapses.

The patients are asked to attend surgical outpatient department after 6 weeks and repeat sonography of abdomen is done to know resolution of the abscess.

All the data obtained from medical records was entered into a proforma questionnaire. At analysis, each item in the questionnaire was analysed separately using the tally method. Statistical analysis was done using mean, averages, Chi-Square and Fisher Exact test to find the significance of proportion wherever applicable using MS Word, Excel and online Graph Pad 3.0 and results compared with the background of recent available literature. Different data presentation methods (Pie charts, bar charts, etc.) were used for all the study parameters and presented in the results.

\section{RESULTS}

Of the total 50 patients screened, 32 (64\%) had PLA and 15 (36\%) had ALA. The major epidemiological findings and clinical features recorded were as follows:

\section{Radiology}

a. Amoebic liver abscess- chest X-ray was abnormal in $44.44 \%$ patients. Ultrasonogram of the abdomen showed right lobe involvement in $88.88 \%$ cases and left lobe involvement in $11.11 \%$. In $77.77 \%$ patients, the abscess was single and in $22.22 \%$ it was multiple. About $77.77 \%$ patients had abscess cavity with size between $5-10 \mathrm{~cm}, 5.55 \%$ with size $<5 \mathrm{~cm}$ and $16.66 \%$ with size $>10 \mathrm{~cm}$.

b. Pyogenic-chest X-ray was abnormal in $68.75 \%$ patients. Ultrasonogram whole abdomen showed right lobe involvement in $75 \%$ cases, left lobe in $12.5 \%$ cases, and both lobes in $12.5 \%$. The abscess was single in $53.12 \%$, multiple in $46.87 \%$ cases. About $37.5 \%$ patients had abscess cavity with size between $5-10 \mathrm{~cm}, 21.87 \%$ with size $<5 \mathrm{~cm}$ and $40.62 \%$ with size $>10 \mathrm{~cm}$.

\section{Blood and Culture Aspirate}

Microbiological confirmation was done in 26 cases out of 32 cases of PLAs. In 4 cases, size of abscesses was less than $2 \mathrm{~cm}$ so it was not done, and in another two cases abscess was very thick and organised so it was not done. Aerobic culture at 370 C for 48 hours revealed growth of E. coli in 7 cases (21.87\%), it was the most common organism cultured in our study.

Klebsiella Pneumoniae and Staphylococcus Aureus were other organism cultured in $4(12.5 \%)$ and $1(3.12 \%)$ case respectively. In $14(43.75 \%)$ cases, culture showed no growth.

Out of 18 cases of ALAs, amoebic serology (IgG antibody) was found to be positive in 16 cases $(88.88 \%)$ and negative in 2 cases $(11.11 \%)$.

\section{Treatment Outcome}

Of 18 patients with ALA, 11 patients were treated with medical or conservative treatment with a success rate of 91.66\%, 4 patients were treated with percutaneous needle aspiration with a success rate of $100 \%, 3$ patients were treated with percutaneous catheter drainage and the success rate of this procedure was $100 \%$. No complications were observed in the present study. None of the patient had to undergo surgical intervention. There was no mortality. Of the 32 patients of PLA, 6 were treated conservatively, 1 was 
treated with percutaneous needle aspiration with the success rate of $100 \%$ after $3^{\text {rd }}$ attempt, and 24 patients were treated with percutaneous catheter drainage with the success rate of $100 \%$. In one case, laparotomy was done for intraperitoneal rupture of a liver abscess, the patient died on 3 rd postoperative day due to multiorgan failure.

\section{DISCUSSION}

In our study, the maximum age incidence for ALA was 21-40 years, with male: female ratio of $2: 1$, [Table 1] which is consistent with previous reports. ${ }^{2-3]}$ Among PLA patients, the maximum age incidence was 41-60 years, with male: female ratio of $1.66: 1$ which is consistent with other reports.[4-5]

\begin{tabular}{|c|c|c|}
\hline Demographic Parameter & PLA & ALA \\
\hline No. of Patients (\%) & $32(64 \%)$ & $18(36 \%)$ \\
\hline Male: Female & $20: 12(1.66: 1)$ & $12: 6(2: 1)$ \\
\hline \multicolumn{3}{|l|}{ Age Wise Distribution \% } \\
\hline $21-40$ & $7(21.87)$ & $14(77.77)$ \\
\hline $41-60$ & $18(56.25)$ & $1(5.55)$ \\
\hline
\end{tabular}

The clinical features observed by us in patients with ALA were abdominal pain (100\%), fever (100\%), jaundice (22.22\%), right upper quadrant tenderness (55.5\%), hepatomegaly $(77.77 \%)$, [Table 2, Figure 1] and are consistent with previous reports.[6] The main clinical features observed in PLA were abdominal pain (90.62\%), fever (93.75\%), jaundice (21.87\%), right upper quadrant tenderness (40.62\%), and hepatomegaly (34.37\%). Several reports have suggested fever and abdominal pain[7,8] being the main presenting feature [Table 2, Figure 1] in PLA.

\begin{tabular}{|c|c|c|}
\hline Symptoms/Signs & PLA (\%) & ALA (\%) \\
\hline Abdominal Pain & $29(90.62)$ & $18(100)$ \\
\hline Fever & $30(93.75)$ & $18(100)$ \\
\hline Weight Loss & $4(12.5)$ & 0 \\
\hline Vomiting & $5(15.62)$ & $5(22.22)$ \\
\hline Diarrhoea & 0 & $4(22.22)$ \\
\hline Cough & $8(25)$ & $5(27.77)$ \\
\hline Jaundice & $7(21.87)$ & $4(22.22)$ \\
\hline $\begin{array}{c}\text { Right Upper Quadrant, } \\
\text { Tenderness }\end{array}$ & $13(40.62)$ & $10(55.55)$ \\
\hline Hepatomegaly & $11(34.37)$ & $14(77.77)$ \\
\hline $\begin{array}{l}\text { Table 2. Comparison of Sign and } \\
\text { Symptoms between Two Groups }\end{array}$ \\
\hline
\end{tabular}

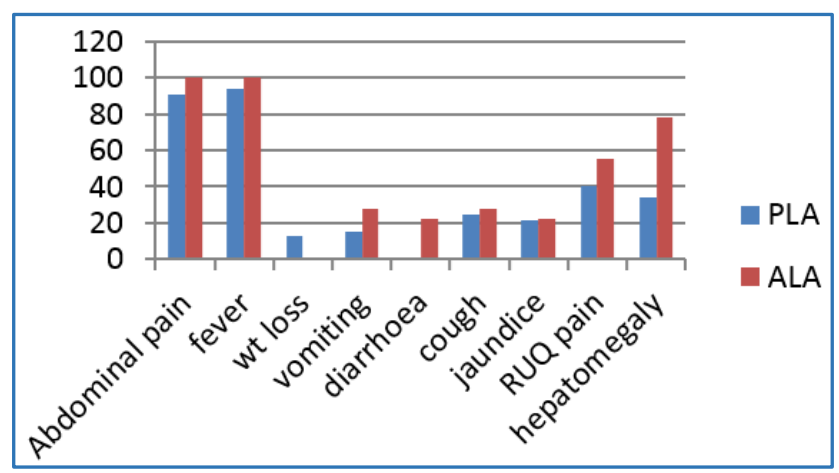

Figure 1. Comparison of Symptoms between Two Groups

In case of ALA, the laboratory investigations revealed leukocytosis (44.44\%), and anaemia (44.44\%). The most marked LFT abnormality was raised alkaline phosphatase (55.55\% cases). Abnormal prothrombin time was observed in $33.33 \%$ cases. Most marked LFT abnormality in patients with PLA was abnormal alkaline phosphatase (62.5\%) and 28.12\% patients had abnormal prothrombin time. About 50\% patients with ALAs and $75 \%$ of PLAs in our study had hypoproteinaemia [Table 3, Figure 2]. In our study, 12.5\% patients with PLA and 60\% patients were diabetic, [Table 3] which is consistent with previous reports.[9] The higher incidence of liver abscesses in diabetics may be due to lower immunity in this patient population.

\begin{tabular}{|c|c|c|}
\hline Investigations & PLA (\%) & ALA (\%) \\
\hline Leukocytosis (>12,000) & $15(46.87)$ & $8(44.44)$ \\
\hline $\mathrm{Hb} \%<10 \mathrm{~g}$ & $23(71.87)$ & $8(44.44)$ \\
\hline Bilirubin $>2.4 \mathrm{mg} / \mathrm{dL}$ & $7(21.87)$ & $4(22.22)$ \\
\hline ALKP Raised & $20(62.5)$ & $10(55.55)$ \\
\hline SGOT Raised & $20(62.5)$ & $13(72.22)$ \\
\hline SGPT Raised & $17(53.12)$ & $10(55.55)$ \\
\hline PT $>16 \mathrm{Sec}$ & $9(28.12)$ & $6(33.33)$ \\
\hline RBS >200 mg/dL & $4(12.5)$ & 0 \\
\hline S Albumin <3 g/dL & $24(75)$ & $9(50)$ \\
\hline \multicolumn{3}{|c|}{ Table 3. Comparison of Laboratory } \\
Investigations between Two Groups \\
\hline
\end{tabular}

ALA-Amoebic liver abscess, PLA-Pyogenic liver abscess.

The most important and accurate diagnostic tool in our study was USG. CECT abdomen was performed in some cases where there was diagnostic confusion. In ALAs, right lobe (88.88\% patients) was most commonly affected. In $77.77 \%$ patients, the abscess was single and in $22.22 \%$ it was multiple. In cases of PLA, the right lobe was involved in $75 \%$ cases and abscess was single in $53.12 \%$ and multiple in $46.87 \%$ cases, which are consistent with previous report.[10,11]

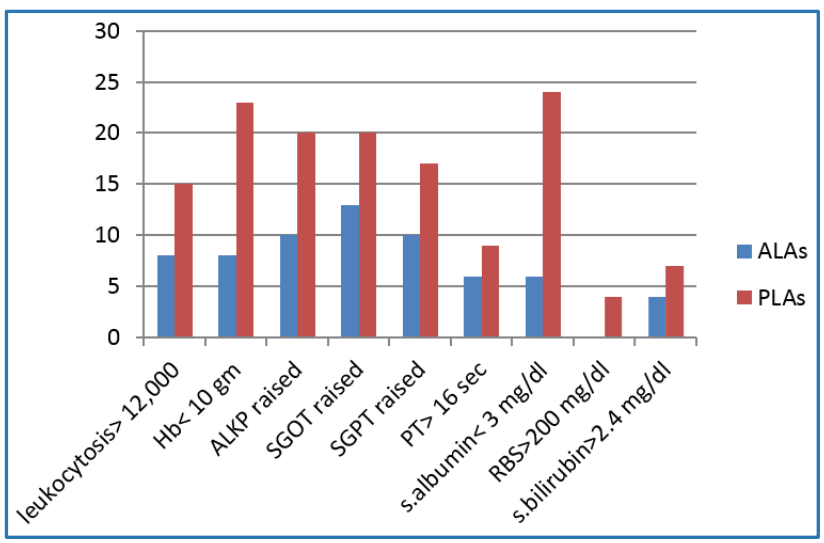

Figure 2. Comparison of Laboratory Investigations between Two Groups

The main aetiology of PLA in our study was E. coli ( $21.87 \%$ cases) as opposed to Klebsiella pneumonia reported by other studies.[12]

In the present study out of 32 cases of PLA, 6 cases (15.75\%) were managed conservatively, percutaneous catheter drainage (Pig tail catheter) was done in 24 cases (75\%). In 1 case $(3.12 \%)$, percutaneous needle aspiration (PNA) was done and in another 1 case (3.12\%) laparotomy was done. 
In the present study, conservative group had a success rate of $100 \%$. There was no mortality in this group. Success rate in the group PNA was also $100 \%$ after 3 rd aspiration with no mortality.

Smith EH et $\mathrm{al}^{13}$ (1974) noticed a success rate of $85 \%$, whereas Giorgio et $\mathrm{al}^{4}$ (1995) observed the success rate of $98.3 \%$ in needle aspiration group. Percutaneous catheter drainage group had success rate of $100 \%$ and $0 \%$ mortality.

Wong et $\mathrm{al}^{14}$ (2009) reported success rate of $94 \%$ in PCD group.

Out of 32 cases of PLA, 1 death was reported. It was a case of intraperitoneal rupture of a liver abscess, who underwent surgical management. The patient died on $3^{\text {rd }}$ post-operative day due to multiorgan failure.

Out of 18 cases of ALA, 11 cases (61.1\%) were managed exclusively by drug therapy, other 3 cases $(16.66 \%)$ were treated by combination of drugs and percutaneous catheter drainage (PCD). Another 4 cases (22.22\%) were treated by percutaneous needle aspiration (PNA). Out of the 12 cases, who were managed conservatively (Drug therapy alone), 11 cases responded very well within 4 to 6 days, with success rate of $91.66 \%$. 4 cases were treated with PNA and 3 cases were drained by pigtail catheter, with the success rate of $100 \%$.

In the present study, needle aspiration after first attempt was successful in 1 case (25\%) only. Rest required multiple attempts which were similar to other report.[15]

None of ALA patients underwent surgical exploration.

\section{CONCLUSION}

Liver abscesses are found to be an important and serious problem in surgical practice in this part of the country, as in other part of the world, because of its obscure nature in the terms of clinical presentation and delayed diagnosis in many instances. Delayed diagnosis and treatment can lead to increased mortality, morbidity, and significant economic impact. Pyogenic liver abscess is found to be common in $5^{\text {th }}$ decade of life, whereas amoebic liver abscess is common in $3^{\text {rd }}$ decade and $4^{\text {th }}$ decade of life. Incidences are little higher in males for both types of abscesses. Early diagnosis and treatment is of paramount importance. A high index of clinical suspicion and repeated and diligent search for unusual signs are required for the diagnosis as most of the bacteriological, haematological and biochemical investigations are nonspecific for intra-abdominal abscesses and give only clues to the infectious process and severity of illness. Ultrasonography is the first radiological procedure to be performed and is sufficient. In the both PLAs and ALAs, most of the lesions are solitary and situated in the right lobe of liver.

E. coli is the most common organism isolated from PLAs

Most of the cases of PLAs are treated with US guided percutaneous catheter drainage. In fact, PCD is a better modality of treatment than needle aspiration as the latter required repeated aspirations and often failed to drain thick viscid pus. Cases with solitary abscess, and smaller than $3 \mathrm{~cm}$ can be treated conservatively. Surgical drainage is mostly entertained in cases of failure of PCD and in case laparotomy is required for any other underlying pathologies like intraperitoneal rupture of abscess. In brief, PCD is the treatment of choice for pyogenic liver abscesses.
For amoebic liver abscesses, drug therapy with Metronidazole remains the treatment of choice for abscesses of size less than $10 \mathrm{~cm}$. Percutaneous needle aspiration is required mostly in cases of Metronidazole resistance and to differentiate between amoebic and pyogenic variety. Catheter drainage is required if there is a large abscess and threatening to rupture.

The mortality is correlated to the severity of the underlying cause, a delayed diagnosis, inadequate drainage, and unsuspected foci of infection within the peritoneal cavity or elsewhere.

\section{REFERENCES}

[1] Ko P. Amoebic liver abscesses. $1^{\text {st }}$ edn. SS Publications, Bombay 1979.

[2] Rubinson HA, Isikoff MB, Hill MC. Diagnostic imaging of hepatic abscesses: a retrospective analysis. Am J Roentgenol 1980;135(4):735-45.

[3] Mathur S, Mohta A, Bhargava N, et al. Clinical profile of amoebic liver abscess. Journal, Indian Academy of Clinical Medicine 2002;3(4):367-73.

[4] Giorgio A, Tarantino L, Mariniello N, et al. Pyogenic liver abscesses: 13 years of experience in percutaneous needle aspiration with US guidance. 1995;195(1).

[5] Karki P, Ansari JA, Koirala S. Liver abscess in the tropics: an experience from Nepal. Southeast Asian J Trop Med Public Health 2004;35(2):425-9.

[6] Gupta RK. Amebic liver abscess: a report of 100 cases. Int Surg 1984;69(3):261-4.

[7] Yoo HM, Kim WH, Shin SK, et al. The changing patterns of liver abscess during the past 20 years--a study of 482 cases. Yonsei Med J 1993;34(4):340-51.

[8] McDonald MI, Corey GR, Gallis HA, et al. Single and multiple pyogenic liver abscesses. Natural history, diagnosis and treatment, with emphasis on percutaneous drainage. Medicine (Baltimore) 1984;63(5):291-302.

[9] Greenstein AJ, Lowenthal D, Hammer GS, et al. Continuing changing patterns of disease in pyogenic liver abscess: a study of 38 patients. Am J Gastroenterol 1984;79(3):217-26.

[10] Ralls PW, Colletti PM, Quinn MF, et al. Sonographic findings in hepatic amebic abscess. Radiology 1982;145(1):123-6.

[11] Mohsen AH, Green ST, Read RC, et al. Liver abscess in adults: ten years experience in a UK centre. QJM 2002;95(12):797-802.

[12] Chan KS, Chen CM, Cheng KC, et al. Pyogenic liver abscess: a retrospective analysis of 107 patients during a 3-year period. Jpn J Infect Dis 2005;58(6):366-8.

[13] Smith EH, Bartrum RJ. Ultrasonically guided percutaneous aspiration of abscesses. Am J Roentgenol Radium Ther Nucl Med 1974;122(2):308-12.

[14] Fong Y, Wong J. Evolution in surgery: influence of minimally invasive approaches on the hepatobiliary surgeon. Surg Infect (Larchmt) 2009;10(5):399-406.

[15] Sharma N, Sharma A, Varma S, et al. Amoebic liver abscess in the medical emergency of a North Indian hospital. BMC Res Notes 2010;3(1):21. 